\title{
Are Expert Opinions Accurate? \\ Panel Data Evidence from the lowa Land Value Survey
}

\author{
Wendong Zhang, Sergio Lence, Todd Kuethe \\ Working Paper 20-WP 612 \\ December 2020 \\ Center for Agricultural and Rural Development \\ lowa State University \\ Ames, lowa 50011-1070 \\ www.card.iastate.edu
}

Wendong Zhang is Assistant Professor, Department of Economics, lowa State University, Ames, IA 50011. E-mail: wdzhang@iastate.edu.

Sergio Lence is Professor, Department of Economics, lowa State University, Ames, IA 50011. E-mail: schlence@iastate.edu.

Todd Kuethe is Associate Professor, Department of Agricultural Economics, Purdue University, West Lafayette, IN 47907. E-mail: tkuethe@purdue.edu.

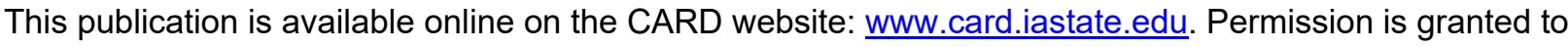
reproduce this information with appropriate attribution to the author and the Center for Agricultural and Rural Development, lowa State University, Ames, lowa 50011-1070.

For questions or comments about the contents of this paper, contact Wendong Zhang, wdzhang@iastate.edu.

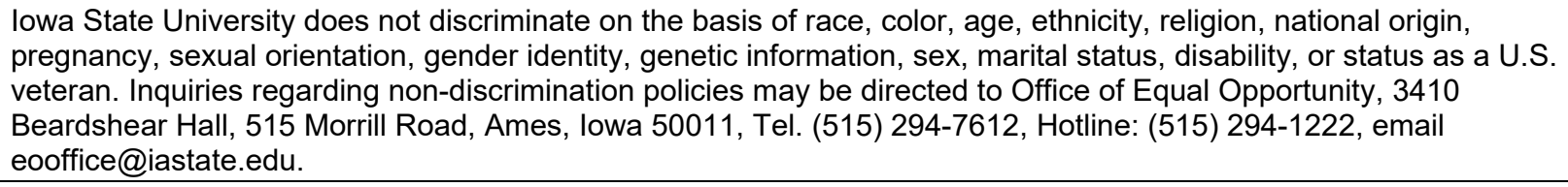




\title{
Are Expert Opinions Accurate? \\ Panel Data Evidence from the Iowa Land Value Survey
}

\author{
Wendong Zhang ${ }^{1}$, Sergio H. Lence ${ }^{2}$, and Todd Kuethe ${ }^{3}$
}

1: Assistant Professor, Department of Economics and Center for Agricultural and Rural Development, Iowa State University, 478C Heady Hall, 518 Farm House Lane, Ames, IA 50011, wdzhang@iastate.edu, 515-294-2536

2: Professor and Marlin Cole Chair of International Agricultural Economics, Department of Economics and Center for Agricultural and Rural Development, Iowa State University, 368E Heady Hall, 518 Farm House Lane, Ames, IA 50011, shlence@iastate.edu, 515-294-8960 3: Associate Professor and Schrader Chair in Farmland Economics, Department of Agricultural Economics, Purdue University, 558 Krannert Hall, 403 W. State St., West Lafayette, IN 47907, tkuethe@purdue.edu, 765-494-4251

\section{Forthcoming at Land Economics}

Acknowledgements: The authors appreciate the feedback from the participants of 2016 NC-1177 meeting in Denver, Colorado, departmental seminars at North Carolina State University and East Carolina University, and the Iowa State University No Free Lunch workshop. The authors thank Nathan Cook for editing assistance. This work was supported in part by the USDA National Institute of Food and Agriculture Hatch project 1010309 and NC-1177. 


\title{
Are Expert Opinions Accurate?
}

\section{Panel Data Evidence from the Iowa Land Value Survey}

\begin{abstract}
(100 words max):
Opinion surveys are the dominant method to gauge U.S. farmland values. However, there lacks a systematic evaluation of how opinions are formulated and adjust over time. Using a panel data of 311 agricultural professionals from the Iowa Land Value Survey from 2005 to 2015, we investigate how surveyed experts update their farmland value estimates. We find that experts almost fully correct their prior "errors" in a single period. Experts' opinions also incorporate most of the prevailing price innovations in one period. Our Bayesian estimation technique simultaneously addresses the non-stationarity of farmland values and the Nickell bias in short dynamic panels.
\end{abstract}

JEL Codes: Q15, Q18

Keywords: (5 max)

Farmland value; expert opinion survey; error-correction model; Bayesian; dynamic panel data model 
Farmland plays a central role in the financial health of the U.S. agricultural sector. Farm real estate makes up over $80 \%$ of the value of the sector's asset base and serves as the primary source of collateral for farm loans (USDA 2019). However, despite farmland's prominent role in the agricultural economy, it remains a challenge to accurately measure the aggregate value of farm real estate. A major reason for this fact is farmland's very low turnover: on average, less than one percent of the stock of U.S. farmland is traded every year (Sherrick and Barry 2003; Zhang and Beek 2016). Zhang, Plastina, and Sawadgo (2018) show that half of the farmland in Iowa has had the same owner for at least twenty years, and many transactions are between family members and thus not arm's length. Another reason is that tracts being traded at any point in time are heterogeneous in quality, location, surrounding land use mix, and sale type (Borchers, Ifft, and Kuethe 2014). In addition, it could take three to nine months between the time the sale is agreed upon and the time it is registered in public county records, which makes it difficult to ascribe a specific date to the corresponding price. Thus, transaction prices are not as informative as desired. Furthermore, there are no consolidated sources of data such as futures markets, CaseShiller residential housing price indices, or Zillow-style farmland sales information, which would allow to quickly gauge representative values for farmland. ${ }^{1}$

In response, institutions such as land grant universities, Federal Reserve banks, professional societies, and the U.S. Department of Agriculture (USDA) conduct periodic surveys of producers or agricultural professionals to measure current market conditions (Kuethe and Ifft 2013). While USDA surveys sample agricultural producers, most other opinion surveys rely on agricultural "experts" or professionals, including real estate agents, rural appraisers, agricultural lenders, farm managers, and county assessors (Zhang and Beek 2016). 
Expert opinion survey estimates of current farmland values are critically important. On the one hand, accurate land market information is difficult to obtain without experts who closely monitor local farmland market movements given the prevalence of non-arm's length transactions, limited farmland sales with time delays in public records, and important differences in farmland parcel characteristics and local market conditions. On the other hand, previous research suggests that on average land value estimates by these knowledgeable experts are comparable with average prices from arm's length farmland sales records (Kuethe and Ifft 2013; Stinn and Duffy 2012; Bigelow, Ifft, and Kuethe 2020). As a result, farmland expert opinion surveys are widely used and attract substantial attention. For example, the 2015 Iowa State University (ISU) Land Value Survey overview was downloaded over 50,000 times and reported by over 50 media outlets. In addition, in many Midwestern states like Iowa, expert opinion surveys often are the only source providing consistent annual land value estimates at the county level.

Interestingly, in spite of the wide use of opinion surveys and the attention they receive, little is known about the behavior of individual experts' opinions over time. In particular, to the best of our knowledge, no study has investigated the degree to which survey estimates reflect respondents' past and current information, and whether and to what extent they adjust or selfcorrect over time. Our study focuses on experts' estimates of current agricultural land values (dollars per acre) year over year. ${ }^{2}$ In particular, we examine the degree to which individual experts adjust their estimates in response to prior errors (i.e., the differences between their stated opinions and prevailing farmland prices), and to changes in market signals. Importantly, these expert estimates are opinions of current land values, not forecasts of future land prices. 
Understanding the behavior of experts in responding to these surveys is critically important for the accuracy of the survey estimates compared to the unobservable prevailing farmland prices. To illustrate this point, consider Figure 1, which is based on expert estimates to the ISU survey for low quality land in South Central Iowa from 1980 through 2019. ${ }^{3}$ This graph depicts an example of the accuracy of average survey estimates in current dollars per acre (panels A and B) and percentage deviations from prevailing prices (panels $\mathrm{C}$ and $\mathrm{D}$ ) when experts fail to fully correct prior errors or do not incorporate all changes in contemporary farmland prices. Blue lines represent observed average expert estimates, whereas other lines represent unobservable prevailing prices implied by different types of imperfections in the dynamics of experts' opinions (which will be discussed in the Model section). The gray line in particular represents the price behavior implied by point estimates obtained in our econometric analysis. The graph shows price dynamics assuming that the observed average expert estimate is $10 \%$ lower than the unobservable prevailing price at the beginning of the period (i.e., year 1980 for panels A and C, and year 2003 for panels B and D). It can be observed that survey estimates could differ by more than $15 \%$ from implied prevailing farmland prices when experts' opinions fail to fully correct prior errors (curves with $-\beta_{y}<1$ and $\beta_{x}<1$ ) or fully incorporate contemporaneous changes in prevailing prices (curves with $\gamma_{x}<1$ ).

We hypothesize that experts self-correct their prior errors. For example, if an expert provides a land value estimate substantially higher than the prevailing price one year, the following year she will tend to adjust current estimates downward. We also expect experts to update current estimates based on other relevant, contemporaneous farmland price signals. To test our hypotheses, we postulate an error-correction model (ECM) to represent the process by which experts update their responses. We conduct our investigation by applying Bayesian 
methods to panel data consisting of 311 experts' individual responses to the annual ISU Land Value Survey from 2005 through 2015. We use multiple different proxies for the unobservable prevailing prices, including county-level average farmland sales prices for $85 \%$ tillable cropland and the median expert opinion to the survey. Our empirical strategy explicitly addresses three important issues relevant to our data, namely, the likely non-stationarity of land values (Lence $2001 ; 2014)$, the fact that "true" land values are not observable, and the Nickell bias (Nickell 1981) associated with short panels like ours. ${ }^{4}$

Overall, our results attest to the accuracy of expert opinions representing prevailing farmland prices. Our analysis suggests that experts' responses conform to an ECM that almost fully corrects prior errors in one period, i.e., experts adjust most of the difference between their prior estimates and the corresponding prior prevailing prices in a single period. We also find that experts' opinions incorporate most of the contemporary innovations in prevailing farmland prices. Interestingly, the speed at which experts self-correct and incorporate new market information depends on the quality of the land for which they furnish estimates. Both selfcorrection and market news assimilation are fastest for high-quality land and slowest for lowquality land. In addition, the long-run elasticity of individual experts' estimates with respect to prevailing prices conforms to the theoretically consistent level of one.

This study makes three important contributions. First, to the best of our knowledge, it provides the first empirical investigation of the behavior of individual farmland value estimates collected through opinion surveys of market experts. The ISU Land Value Survey analyzed is one of the most widely followed surveys of land values. Second, the empirical results provide strong evidence that experts' estimates conform to an ECM in which they quickly self-correct prior errors and almost instantaneously incorporate changes in market price signals. However, 
experts' estimates are found to be less informative and noisier for low-quality land, suggesting that more trust should be put in the ISU Land Value Survey for high-quality land values than for low-quality land values. Finally, the proposed methodological framework, based on the Bayesian estimation of an ECM, provides a useful approach to analyze the behavior of individual responses in panel data from surveys. In particular, it should prove valuable for surveys involving non-stationary variables, unobservable "true" values, or short panels (where correcting for Nickell bias is critical).

\section{Model}

The farmland value surveys we are concerned about elicit experts' opinions about the current value of farm real estate of a given quality within a certain market boundary (such as a county). Each expert $i$ reports her individual subjective value for the current time period $t$ and her primary county $c(i)$, denoted $Y_{i, t}$, for the unobservable prevailing price of farmland $X_{c(i), t .}$ Variable $X_{c(i), t}$ could also be interpreted as the unobservable true market value; for brevity, we will refer to $X_{c(i), t}$ as the prevailing price hereafter. Taking logarithms $y_{i, t} \equiv \ln \left(Y_{i, t}\right)$ and $x_{c(i), t} \equiv \ln \left(X_{c(i), t}\right)$, if market boundaries are defined such that farmland is homogenous in quality and all other attributes, free and complete information would yield

$$
y_{i, t}=x_{c(i), t}+\varepsilon_{i, t} ; \quad \forall i, t
$$

with essentially zero error $\varepsilon_{i, t}$. In our application, multiple experts provide estimates for the same county, but each individual expert has only one primary county. As a result, $x_{c(i), t}=x_{c(j), t}=x_{c, t}$ for experts $i$ and $j$ who report for the same county. 
To analyze the process by which experts update their subjective valuations, rearrange equation (1) after adding and subtracting lagged land price terms, so as to obtain the ECM

$$
\Delta y_{i, t}=-\left(y_{i, t-1}-x_{c(i), t-1}\right)+\Delta x_{c(i), t}+\varepsilon i, t ; \quad \forall i, t,
$$

where $\Delta y_{i, t} \equiv y_{i, t}-y_{i, t-1}$, and $\Delta x_{c(i), t} \equiv x_{c(i), t}-x_{c(i), t-1}$. According to this ECM, for an expert's subjective valuation in year $t$ to be identical to the corresponding prevailing price, the change in the individual's valuation from the previous year (i.e., $\left.\Delta y_{i, t}\right)$ must consist of two parts. The first component, $-\left(y_{i, t-1}-x_{c(i), t-1}\right)$, is an error-correction term that fully corrects for any difference between the expert's prior subjective valuation and the prior prevailing price. The second component, $\Delta x_{c(i), t}$, is the full contemporaneous innovation in the prevailing price, i.e., the change in the prevailing price over the past year. Thus, an expert's subjective valuation will be identical to the prevailing price in year $t$ if the expert: $(a)$ fully corrects for the previous year's difference between her subjective valuation and the prevailing price at the time; and, $(b)$ fully incorporates the innovation in the prevailing price.

Our analysis is based on ECM (2). An expert's error-correction behavior may be driven by self-pride, professional ethic, peer pressure, or "herd behavior," in which the expert leverages the collective decisions or information exhibited by other experts in the previous period (Banerjee 1992). For estimation and hypothesis testing purposes, we generalize ECM (2) by incorporating coefficients multiplying explanatory variables, and decomposing the error into an intercept $(\alpha)$ and a zero-mean residual $\left(e_{i, t}\right)$, so as to yield the regression

$$
\Delta y_{i, t}=\alpha+\beta_{y} y_{i, t-1}+\beta_{x} x_{c(i), t-1}+\gamma_{x} \Delta x_{c(i), t}+e_{i, t} ; \quad \forall i, t .
$$


Regression (3) collapses into ECM (2) when coefficients satisfy the conditions $\left\{\alpha=0,-\beta_{y}=\beta_{x}\right.$ $\left.=\gamma_{x}=1\right\}$. Thus, such conditions imply that data are consistent with the ideal scenario where experts' estimates fully reflect prevailing prices. More generally, according to regression (3), the innovation in the $i^{\text {th }}$ expert's estimate incorporates a fixed amount of $\alpha$, which is identical for all experts, and $100 \gamma_{x}$ percent of the innovation in the prevailing price $\Delta x_{c(i), t .}$ In addition, since $\left(\beta_{y}\right.$ $\left.y_{i, t-1}+\beta_{x} x_{c(i), t-1}\right)=\left[\beta_{y}\left(y_{i, t-1}-x_{c(i), t-1}\right)+\left(\beta_{y}-\beta_{x}\right) x_{c(i), t-1}\right]$, the innovation in the expert's response "corrects" $100 \beta_{y}$ percent of the lagged error $\left(y_{i, t-1}-x_{c(i), t-1}\right)$ in a single period, and it includes $\left(\beta_{y}\right.$ $-\beta_{x}$ ) of the previous prevailing price $x_{c(i), t-1}$

Figure 1, which was briefly discussed in the introduction, illustrates the implications of parameters $\beta_{y}, \beta_{x}$, and $\gamma_{x}$ for unobservable prevailing prices $(x)$ given an actual series of observed average expert estimates $(y)$ if there were no errors $\left(e_{i, t}=0 \forall i, t\right)$, which is represented by the blue line. For simplicity, the graph is drawn by setting $\alpha=0$, and the observed average expert estimate $10 \%$ lower than the unobservable prevailing price at the beginning of the period (i.e., year 1980 for panels A and C, and year 2003 for panels B and D). The orange line represents the unobservable prevailing prices implied by the observed estimates if the experts' behavior is characterized by $\left\{-\beta_{y}=\beta_{x}=0.5, \gamma_{x}=1\right\}$. In this instance, the gap between the prevailing price and the average expert estimate closes by $50 \%$ per period, and it becomes negligible after a few periods because the expert estimate fully incorporates the innovations in the prevailing price $\left\{\gamma_{x}\right.$ $=1 \mathrm{f}$. The parameterization underlying the green line differs from the one for the orange line only in that just half of the prevailing price innovation is incorporated in each period $\left\{\gamma_{x}=0.5\right\}$. It can be seen that the smaller magnitude of parameter $\gamma_{x}$ has a profound impact on implied prevailing prices, and that it takes many periods without prevailing price innovations to yield a stable 
negligible gap between average expert estimates and prevailing prices. Figure 1 also shows that the situation where $-\beta_{y}$ is greater than $\beta_{x}$, represented by the orange line, results in a consistently positive gap between average expert estimates and prevailing prices. Such gap is negative in the opposite scenario with $-\beta_{y}<\beta_{x}$, which is omitted to avoid clutter.

In practice, there is no widely accepted objective measure of the value for a representative tract of farmland. A number of attributes of farmland and its market help explain this stylized fact. Farmland is heterogeneous in quality, percent tillable, use, and extent of urban influence, which renders the sale price of a particular tract quite specific and difficult to extrapolate to other tracts. Also, farmland available for sale is fairly limited, especially for arm's length transactions, and farmland markets tend to be localized. Furthermore, actual farmland sales are lengthy processes, often taking three to nine months from the time a price is agreed upon until the transaction is posted in public county assessor records. As a result, assigning a date to a sale price is not a trivial task.

For the aforementioned reasons, it is useful to allow for systematic biases in experts' opinions. To consider this generalization, let $Y_{i, t}=A_{i} X_{c(i), t}$ for $A_{i}>0$, with $A_{i}<(>) 1$ meaning that the $i^{\text {th }}$ expert consistently under-reports (over-reports) the prevailing price. To incorporate this feature, regression (3) is generalized as the experts' fixed-effects (FE) ECM regression (4):

$$
\Delta y_{i, t}=\alpha_{i}+\beta_{y} y_{i, t-1}+\beta_{x} x_{c(i), t-1}+\gamma_{x} \Delta x_{c(i), t}+e_{i, t}
$$

where $\alpha_{i}=-\beta_{y} \ln \left(A_{i}\right)$ represents the extent to which the $i^{\text {th }}$ expert's response consistently underor over-estimates prevailing prices. 


\section{Experts' Estimates in the Long Run, and Their Short-Term Dynamics}

Ideally, regression (4) should satisfy conditions $\left\{-\beta_{y}=\beta_{x}=\gamma_{x}=1\right\}$, so that the innovation on the expert's response $\left(\Delta y_{i, t}\right)$ fully corrects her previous error $\left(y_{i, t-1}-x_{c(i), t-1}\right)$ in a single period, and fully incorporates the innovation in the prevailing price $\left(\Delta x_{c(i), t)}\right.$. In reality, however, an expert may take a longer time to revise a previous estimate, in which case lagged innovations in the expert's estimate or in the prevailing price may explain the current innovation. To allow for this possibility, we expand regression (4) as

$$
\Delta y_{i, t}=\alpha_{i}+\beta_{y} y_{i, t-1}+\beta_{x} x_{c(i), t-1}+\gamma_{x} \Delta x_{c(i), t}+\sum_{n_{y}=1}^{N_{y}} \delta_{y, n_{y}} \Delta y_{i, t-n_{y}}+\sum_{n_{x}=1}^{N_{x}} \delta_{x, n_{x}} \Delta x_{c(i), t-n_{x}}+e_{i, t},
$$

where $\delta$ s are lagged innovations' coefficients.

A nice feature of the ECM is that it is straightforward to infer the "long-run" or "equilibrium" relationship between experts' estimates and the set of explanatory variables (i.e., the relationship when neither variable has a tendency to change). To illustrate, consider ECM (5) - by setting first-difference terms and residuals equal to zero, dropping time subscripts, and rearranging, we obtain equilibrium relationship (6) in logarithms

$$
y_{i}=-\alpha_{i} / \beta_{y}-\beta_{x} / \beta_{y} x_{c(i)}
$$

which is equivalent to $Y_{i}=A_{i} X_{c(i)}^{-\beta_{l} / \beta_{y}}$ in levels. According to these expressions, $-\beta_{x} / \beta_{y}$ is the long-run elasticity of experts' estimates with respect to prevailing land prices. Ideally, such elasticity should be characterized by $-\beta_{x} / \beta_{y}=1$, in which case a $1 \%$ change in the prevailing price translates into a $1 \%$ change of the same sign in the expert estimate in the long run. If 
$-\beta_{x} / \beta_{y}>1$, in the long run the expert estimate will increase/decrease by more than $1 \%$ in response to a $1 \%$ increase/decrease in the prevailing price. Ceteris paribus, long-run expert estimates will be greater (smaller) than prevailing prices when $-\beta_{x} / \beta_{y}>1$. Figure 1 illustrates scenarios with $-\beta_{x} / \beta_{y}=1$ and $-\beta_{x} / \beta_{y}>1$.

Note also that if the expert's estimate at time $(t-1)$ satisfies equilibrium condition (6), the time- $t$ expected expert innovation from ECM (5) consists of

$$
\Delta y_{i, t}=\gamma_{x} \Delta x_{c(i), t}+\sum_{n_{y}=1}^{N_{y}} \delta_{y, n_{y}} \Delta y_{i, t-n_{y}}+\sum_{n_{x}=1}^{N_{x}} \delta_{x, n_{x}} \Delta x_{c(i), t-n_{x}} .
$$

Thus, the first-difference (i.e., innovation) terms on the right-hand side of ECM (5) represent purely short-term dynamics in expert's estimates.

\section{Drivers of Experts' Estimates Other Than Land Values}

As already emphasized, a key feature of farmland markets is that they are quite thin, and the traded asset is heterogeneous. Hence, we can reasonably hypothesize that experts may resort to sources of information other than actual sales to enhance the quality of their estimated values. If this is the case, interest rates and farmland rental rates are the most likely candidate sources of additional information. The asset capitalization model posits that there should be a long-run relationship between land values $(X)$, land rental rates (RENT), and the interest rate (INTEREST). More specifically, according to the asset capitalization model, there is a long-run relationship $X=$ RENT/INTEREST in levels, or $x=$ rent - interest in logarithms, which implies a long-run relationship among experts' estimates, rental rates, and interest rates, if the asset capitalization model holds. However, if the expert knew the prevailing land price, neither rental rates nor 
interest rates (or any other related variables) should provide relevant information to experts when they respond to a land value survey.

To test the degree to which expert estimates are affected by rental rates and interest rates conditional on land values, we fit the following ECM regression:

$$
\begin{aligned}
& \Delta y_{i, t}=\alpha_{i}+\beta_{y} y_{i, t-1}+\beta_{x} x_{c(i), t-1}+\gamma_{x} \Delta x_{c(i), t}+\sum_{n_{y}=1}^{N_{y}} \delta_{y, n_{y}} \Delta y_{i, t-n_{y}}+\sum_{n_{x}=1}^{N_{x}} \delta_{x, n_{x}} \Delta x_{c(i), t-n_{x}} \\
& +\beta_{\text {rent }} \perp \text { rent }_{c(i), t-1}+\beta_{\text {interest }} \perp \text { interest }_{t-1}+\gamma_{\text {rent }} \perp \Delta \text { rent }_{c(i), t}+\gamma_{\text {interest }} \perp \Delta \text { interest }_{t}
\end{aligned}
$$

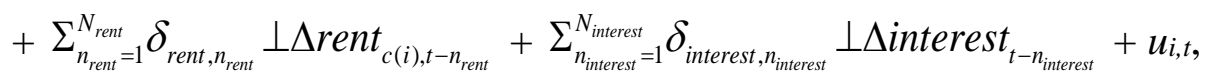

where $\perp w$ is the component of variable $w$ orthogonal to the set of regressors in ECM (5); and $\Delta \operatorname{rent}_{c(i), t}$ and $\Delta$ interest $_{t}$ denote the first differences (i.e., innovations) in the logarithms of rental rates and interest rates, respectively. ${ }^{5}$ If the $\perp w$ regressors in ECM (8) are significant, then rental rates and/or interest rates have an impact on expert responses above and beyond the effect they may have on prevailing land prices. ${ }^{6,7}$

Finding that coefficients associated with the orthogonal components of land rents or interest rates are different from zero suggests that experts use information on land rents and interest rates to supplement their knowledge about land values. If experts felt sufficiently confident about their knowledge about land values when answering a survey, their responses (conditional on such values) should not be affected by rental rates or interest rates. ${ }^{8}$ 
Land quality represents a critical source of heterogeneity that makes prevailing land price signals for high-quality land more salient than for lower quality land. Heterogeneity is important for several reasons. First, experts tend to have a better understanding of productivity for high-quality land relative to lower quality land (Zhang and Duffy, 2017). Second, high-quality farmland is typically devoted to crop production, whereas low-quality farmland may include recreational grounds, pasture, or timberland. Third, there is more variation in price per soil quality index for low-quality land than for higher quality land (Zhang and Duffy 2017). Finally, higher quality land tends to be more heavily promoted at land auctions, usually receives more local news coverage, and is more frequently discussed among producers, landowners, and agricultural professionals. As a result, it is natural to hypothesize that experts have better knowledge of prevailing prices for high-quality land than that for low-quality land.

Econometrically, this would suggest that information about prevailing prices for lowquality land $x_{c(i), t}^{L}$ contains more noise. Thus, for low-quality land, the coefficients in equation (4) may be more likely to deviate from the ideal conditions $\left\{\alpha_{i}=0,-\beta_{y}=\beta_{x}=\gamma_{x}=1\right\}$, and the corresponding error term $e_{i, t}^{L}$ may have a larger variance. More specifically, we hypothesize that the magnitude of the under- or over-estimation by experts for low-quality land is more noticeable due to noisier market signals than that for high-quality land. The magnitude for $\left\{-\beta_{y}, \beta_{x}, \gamma_{x}\right\}$ is hypothesized to be smaller for low-quality land as well, due to the greater noise.

With noisier market signals, it is reasonable to expect experts to rely more on additional information, such as rental rates and interest rates, when providing value estimates for lower quality land. Thus, we also hypothesize that the coefficients associated with the orthogonal components of land rents or interest rates $\left\{\beta_{\text {rent }}, \beta_{\text {interest }}, \gamma_{\text {rent }}, \gamma_{\text {interest }}, \delta_{\text {rent }}, \delta_{\text {interest }}\right\}$ in regression (8) are larger in magnitude for low-quality land than for high-quality land. 


\section{Data}

Table 1 reports summary statistics for key variables used in our study. Our main data source consists of individual expert responses to the annual ISU Land Value Survey from November 2005 through 2015. Figure 2 shows a sample of the survey questions sent to experts in November 2015. In early November, the survey solicits experts for their opinions about land values per acre for high-, medium-, and low-quality land for average-sized farms in their primary county as of November $1^{\text {st }}$ (i.e., a few days earlier). That is, expert opinions essentially represent estimates of current land values, as opposed to forecasts of future values. Experts are mainly agricultural professionals knowledgeable about land markets, such as farm managers, rural appraisers, agricultural lenders, real estate brokers, and county assessors. Previous research has shown that on average these expert estimates from this survey are similar to farmland sales prices (Stinn and Duffy 2012).

The data structure consists of an unbalanced panel, because not every expert participates in the survey each year. To avoid having too few time series observations for any individual, in our regressions we only use estimates $\left(y_{i, t}\right)$ from experts who participated in at least seven of the eleven surveys. Thus, the study involves responses from 311 experts, $36 \%$ of whom participated in all surveys during the period, and $12 \%, 16 \%, 18 \%$, and $18 \%$ of whom provided responses in ten, nine, eight, and seven of the surveys, respectively.

In general, there were more experts in northern and central Iowa than in southern Iowa, which is consistent with the distribution of farms and tillable farmland across the state. In particular, the Northwest Iowa and Northeast Iowa crop reporting districts (CRDs) each comprises more than $14 \%$ of experts, whereas the East-Central Iowa and Southern Iowa CRDs 
each accounts for less than $10 \%$ of experts. The vast majority $(82 \%)$ of experts gave land value estimates for only one county, and only $7 \%$ of experts furnished estimates for more than three counties. For consistency, if an expert provided land value estimates for more than one county, only estimates for her primary county were used for our analysis.

As discussed previously, there is no objective, readily available measure of the prevailing land price $\left(X_{t}\right)$, especially at the county level. Given this limitation, our analysis is performed using the following proxies for the unobservable prevailing price corresponding to expert $i$ 's estimates: (a) county median estimate (the median of estimates from all experts for land of a particular quality in expert $i$ 's county); (b) CRD median estimate (the median of the county median estimates defined in ( $a$ ) for expert $i$ 's CRD); (c) Farm Credit Services (FCS) county average sales price based on arm's length transactions of cropland parcels that are at least $85 \%$ tillable; $(d)$ FCS CRD median price (the median of the FCS county prices defined in $(c)$ for expert $i$ 's CRD); (e) Realtors Land Institute (RLI) CRD average value; and (f) ISU CRD average value.

To extract as much information as possible from the data, the medians for cases $(a)$ and (b) are constructed using the entire set of experts (i.e., not restricted to experts who participated in at least seven of the eleven surveys). The rationale for using medians instead of averages to construct the prevailing price proxies is to avoid having the latter unduly affected by outliers. This concern is especially strong at the county level because the number of experts for any given county is relatively small, which makes the average more vulnerable to extreme values. The number of expert responses used to construct county median land values ranged from a low of three to a high of eleven, with an average of seven per county. 
The FCS county price series $(c)$ consists of the average sales prices for all arm's length transactions for cropland that is at least $85 \%$ tillable, regardless of whether sales are financed by FCS or not (Zhang 2016). Unlike the other series, FCS series $(c)$ and $(d)$ do not discriminate among high-, medium-, and low-quality land. However, the FCS series most likely involve larger shares of high-quality land than low-quality land because of the $85 \%$ tillable restriction. Furthermore, the FCS sales prices series are not published or publicized, so it is more difficult for respondents to access when they do not work at the Farm Credit System banks.

Series $(b),(d),(e)$, and $(f)$ are constructed to represent CRD-level values. Given that the ISU survey asks for county-level estimates, using CRD-level series to proxy prevailing prices might seem problematic. However, CRD-level series are relevant for at least three reasons. First, CRD-level variables are subject to less sample variability because they are based on larger samples (e.g., on average, CRD medians rely on 36 expert responses, compared to just seven responses for county medians). Second, experts are likely to consider information on land values beyond their own counties due to the thinness and spatial connectedness of farmland markets. Third, model (6) accommodates this scenario if county-level estimates are proportional to CRDlevel prevailing prices.

The RLI CRD series $(e)$ consists of the September values for tillable cropland reported by the RLI Iowa Chapter Survey (Hansen 2018). Notably, RLI survey directly asks for CRD- rather than county-level estimates, and it mainly surveys farm managers, who also represent $15 \%$ of the ISU surveyed experts. Finally, the ISU CRD series $(f)$ is the average of the individual expert primary county estimates across all respondents to the ISU survey with primary counties located within a particular CRD. This average is released every year during mid-December and is meant to represent the land value as of November 1 for that particular year. Series $(f)$ and $(b)$ are 
different because $(f)$ is the simple average across all raw responses, whereas $(b)$ is the median expert response.

The land rent is the average cash rent at the CRD and county level published every May from the ISU Cash Rent Survey (Plastina 2018), a producer and landowner opinion survey. Finally, the interest rate is the state-average interest rate on farm real estate published each October from the Federal Reserve Bank of Chicago quarterly Land Values and Credit Conditions Survey (Oppedahl 2018).

\section{Econometric Estimation}

We estimate the proposed ECM using Bayesian methods. A comprehensive description of the methods is somewhat involved; hence, for the sake of clarity, in the remainder of this section we present an overview, relegating details to Appendix A.

The Bayesian estimation allows us to avoid a number of issues that would arise if we were to perform inference under classical methods for the case of non-stationary farmland values. ${ }^{9}$ Regardless of whether farmland values are stationary or not, given the proposed ECM structure, Bayesian methods allow us to estimate the parameters' posterior distributions in a straightforward manner, conditional on the initial set of observations of the model variables (Lancaster 2002). Such posterior distributions can then be employed to test various hypotheses of interest, as desired. ${ }^{10}$ Furthermore, as explained next, the proposed approach also allows us to address two important issues with the present data, namely, the Nickell bias (Nickell 1981) and errors-in-variables.

The Nickell bias arises when estimating FE panel models with lagged dependent variables using standard methods (e.g., ordinary least squares and standard maximum 
likelihood), because in that case the coefficient of the lagged dependent variable is biased (Nickell 1981). The Nickell bias is negative if the first-order autoregressive coefficient is positive, and it is worse for shorter panels (Nickell 1981). To deal with the Nickell bias, we resort to the method recently developed by Dhaene and Jochmans (2016), who generalize Lancaster's (2002) orthogonal reparameterization approach. ${ }^{11}$ By appropriately reparemeterizing the FEs, this approach allows one to estimate the "common parameters" (i.e., parameters other than the FEs) independently of the FEs and circumvent the Nickell bias.

The errors-in-variables problem is the result of using proxies for prevailing land prices, which are unobservable. The errors in the proxies are incorporated into the estimation by adopting the classical errors-in-variables assumption that the $c$ th county proxy variable $\left(\breve{x}_{c, t}\right)$ is a noisy but unbiased measure of the unobservable prevailing price $\left(x_{c, t}\right)$ (Fuller 1987): ${ }^{12}$

$$
\breve{x}_{c, t}=x_{c, t}+\varepsilon_{c, t}
$$

where $x_{c, t} \sim \mathrm{N}\left(\mu_{x}, \sigma_{x}^{2}\right), \varepsilon_{c, t} \sim \mathrm{N}\left(0, \sigma_{\varepsilon}^{2}\right)$ and independent of $x_{c, t}$, and $\mathrm{N}\left(\mu, \sigma^{2}\right)$ denotes the normal distribution with mean $\mu$ and variance $\sigma^{2}$.

Following the recommendations by Gelman (https://github.com/stan-dev/stan/wiki/PriorChoice-Recommendations), we adopt weakly informative priors for the Bayesian estimation. Priors are assumed to be distributed as Student's t for the regression coefficients, and as halfCauchy for the standard deviations. All prior distributions are parameterized so that priors have standard deviations at least 10 times larger than the corresponding posteriors, ensuring that posteriors are driven mostly by the data rather than the priors. 
Estimation is performed using RStan (https://cran.rproject.org/web/packages/rstan/vignettes/rstan.html), the $\mathrm{R}$ interface to Stan in the R version 3.5.1 programming language and software environment (https://www.r-project.org). Stan 2.19.0 is employed to implement Hamiltonian Monte Carlo (HMC) sampling with the No-U-Turn sampler (Stan Development Team 2019). For each of the estimated regressions, the HMC procedure is conducted using four chains, each of them consisting of 2,000 iterations. The first 1,000 iterations of each chain are discarded as a burn-in period. The Gelman-Rubin test (Gelman and Rubin 1992) is then applied to check the convergence of the remaining part of the chains for each of the parameters.

Upon convergence, the 4,000 simulated values for each parameter are taken to be draws from the parameter's posterior marginal distribution. The 4,000 sets of simulated parameters are also used to obtain the posterior distributions for some values of interest, such as the long-run elasticity $-\beta_{x} / \beta_{y}$, which should equal one if $\beta_{y}=-\beta_{x}$, as hypothesized by the restricted ECM.

\section{Results and Discussion}

Estimation results are summarized in tables 2 through 5. For each parameter (or function of parameters) of interest, the tables report the mean, standard deviation (within parentheses), and the $2.5 \%$ and $97.5 \%$ quantiles (within brackets) of the estimated posterior distribution. Such quantiles constitute the upper and lower bounds of the 95\% credible intervals (CIs) for the respective parameters. CIs are the Bayesian analogs of confidence intervals in classical methods. The tables also report the posterior mean, standard deviation, and 95\% CI for the Bayesian $\mathrm{R}^{2}$ of each estimated model. ${ }^{13}$ Gelman-Rubin test statistics are not reported because they are smaller than 1.005 in all instances, which provides strong evidence of convergence. 
Table 2 presents estimation results for regression (5) (i.e., the basic ECM with no FEs). Columns I-III showcase results for high-, medium-, and low-quality land using prevailing prices proxied by experts' median subjective valuations at the county level, whereas columns IV-VI present results at the CRD level. The error-correction coefficients ( $\beta_{y}$ and $\beta_{x}$ ) exhibit the expected signs, because their posterior means and virtually their entire posterior distributions are on the expected side of zero. The posterior means of $\beta_{y}$ and $\beta_{x}$ indicate that experts' opinions only correct about $20 \%$ of the previous error in a single year. The posterior mean of the coefficient for the contemporaneous prevailing price innovation $\left(\gamma_{x}\right)$ is small. However, this coefficient is significantly negative, in the sense that the $95 \%$ CI consists entirely of negative numbers, which is the opposite of what would be expected. The coefficients on lagged innovations ( $\delta_{y}$ and $\delta_{x}$ ) are also significantly different from zero, suggesting relatively strong short-term dynamics.

Overall, estimates of the ECM with no FEs reported in table 2 are substantially different from the ideal scenario in which experts' opinions fully reflect prevailing prices (i.e., $\left\{\alpha=0,-\beta_{y}\right.$ $\left.\left.=\beta_{x}=\gamma_{x}=1\right\}\right)$. This is likely the result of the single, common intercept imposed by this model, which does not reflect the fact that experts may possess different subjective beliefs about prevailing land prices, due to their heterogeneous knowledge, experience, and perceptions of the market and other relevant signals. When considered in light of the implied prevailing price dynamics illustrated in Figure 1, the estimated ECM with no FEs --with low $-\beta_{y}$ and $\beta_{x}$, and negative $\gamma_{x}$-- seems to provide a very poor representation of the behavior of experts' opinions. Therefore, the remainder of the discussion focuses on results for the FE ECM regressions.

Table 3 presents results for our preferred FE ECMs. For example, the first column shows that for high-quality land, taking the county-level median as a proxy for the prevailing price, and 
holding other characteristics constant, an individual expert's opinion will correct about $88 \%$ of the error she made in the immediately preceding year, and incorporate about $87 \%$ of the contemporaneous prevailing price innovation. Furthermore, the expert's opinion exhibits no short-term dynamics, as the coefficients for the lagged innovations have very small posterior means and $95 \%$ CIs that include zero. ${ }^{14}$

The estimates reported in table 3 provide strong evidence that experts' opinions conform to an ECM with almost instantaneous adjustments, where experts not only promptly self-correct prior errors but also almost instantaneously fully incorporate innovations in the prevailing price. In addition, there is no evidence of short-term dynamics in experts' opinions, as the $95 \%$ CIs for coefficients $\delta_{y}$ and $\delta_{x}$ include zero. Importantly, the long-run elasticity of experts' responses with respect to the prevailing price conforms to the theoretically consistent coefficient of one for all specifications shown in table 3 . Not only do long-run elasticities have posterior means very close to one, but also all of their $95 \%$ CIs contain the unit value.

Even though the posterior distributions of the long-run elasticities are very similar across land qualities, table 3 reveals a clear pattern regarding the speed at which experts self-correct and incorporate new market information depending on the quality of the land for which they furnish opinions. For example, the posterior mean speed at which an expert corrects her prior error falls from about $90 \%$ for high-quality land, to around $84 \%$ and $66 \%$ for medium- and low-quality land, respectively. Similarly, parameter $\gamma_{x}^{\prime}$ 's posterior means indicate that expert opinions incorporate about $87 \%$ of the contemporaneous innovation in the prevailing price for highquality land, compared to $84 \%$ and $77 \%$ for medium- and low-quality land. In addition, the model explains a larger proportion of the variance of predicted values for higher quality land, as demonstrated by the Bayesian $\mathrm{R}^{2} \mathrm{~s}$ : posterior means for high-, medium, and low-quality land are 
respectively $0.853,0.828$, and 0.758 , and the $95 \%$ CIs do not overlap. Similarly, residual standard deviations have posterior means that become larger as land quality worsens (increasing from 0.151 for high quality to 0.218 for low quality), and non-overlapping $95 \%$ CIs.

The results suggest that land market signals are more informative in explaining experts' land value estimates for high-quality land than for lower quality land. Possible reasons for this fact are that low-quality land exhibits greater heterogeneity in quality and percent tillable, larger variation in price per soil quality index, and fewer high-profile auction sales, resulting in less public attention when compared to high-quality land. It is also plausible that experts are more likely to remember higher-priced land auction sales. Previous research has found a wider range for both the productivity index and the price paid productivity index unit associated with lower quality land (Zhang and Duffy 2017).

Table 4 presents a robustness check incorporating orthogonal components of interest rates and cash rents into the key specification (i.e., regression (8)). Table 4 shows that most of the $95 \%$ CIs for the cash rent and interest rate coefficients do not include zero, indicating that they are statistically significant. Overall, however, the impact of cash rents and the interest rate on experts' opinions is relatively small, because the posterior distributions of the residual standard deviation and the Bayesian $\mathrm{R}^{2}$ are very similar to their counterparts in table 3 .

The effects of cash rents and the interest rate tend to be larger for medium- and lowquality land compared to those for high-quality land. This is consistent with the notion that experts use information about cash rents and interest rates to supplement and refine their opinions, especially when land market signals are noisier (as is the case with lower quality land). Furthermore, experts seem to respond more strongly to information about the interest rate compared to cash rents. This finding could be due to the fact that interest rates are more uniform 
across the nation and thus arguably more salient signals to all experts, whereas cash rent data are more heterogeneous and stickier in nature. Leases often last longer than one year; hence, cash rents may take some time to reflect changes in crop and livestock market fundamentals driving innovations in land values.

Finally, we present robustness checks to assess the stability of our results based on alternative proxies for prevailing prices, instead of the median expert response used in the main specification. First, table 5 presents estimates obtained by proxying the true land values with the county-level average sales price for arm's length transactions of $85 \%$ tillable cropland collected by FCS. A comparison of tables 3 and 5 shows that our main results regarding experts' errorcorrection behavior remain qualitatively similar. However, the experts' opinions incorporate a significantly smaller share of the contemporaneous innovations in the prevailing price, long-run elasticities are smaller than the theoretically consistent value of one, and there are significant lagged dynamics. ${ }^{15}$ Possible reasons for these results are that the FCS prices correspond to $85 \%$ tillable cropland, which may mostly comprise high-quality tracts; they are only available for the relatively high price years from 2008 to 2015; and they are an average of the sales prices over the year, rather than a value at a specific point in time (i.e., November $1^{\text {st }}$ for the ISU survey).

Finally, we report in Appendix C several robustness checks using land value estimates from two publicly released surveys as proxies for the prevailing prices, or using different configurations of our estimation samples. Table $\mathrm{C}$ uses publicly released CRD average values from RLI and ISU surveys. Overall, a comparison of table 3 with this table shows that our main results regarding experts' error-correction behavior remain qualitatively similar. Specifically, experts self-correct most of the errors in the preceding year in one period, and incorporate most of the contemporaneous innovation in the prevailing price. Further, the error-correction behavior 
and the incorporation of the contemporaneous innovation are more pronounced for high-quality land than for lower quality land. Our results are also robust when using subsamples based on types of respondents and number of experts used to construct the median expert estimates (see tables C.2 and C.3).

\section{Conclusions}

Using a panel data of 311 agricultural experts responding to the ISU Land Value Survey from 2005 through 2015, we develop an ECM model to examine how experts update their responses over time. This updating behavior is critically important for the accuracy of expert opinion surveys like the Iowa survey, as it can be severely reduced if experts fail to fully correct prior errors and do not fully adjust their estimates to incorporate innovations in prevailing land prices.

Our main results provide strong evidence that experts' survey responses exhibit errorcorrection behavior with almost instantaneous adjustments in just one period. These results are robust to alternative measures of the prevailing farmland prices, including the county-level average sales prices for arm's length transactions of cropland parcels that are at least $85 \%$ tillable. We also find that the speed at which experts correct prior errors and incorporate contemporaneous innovations is faster for high-quality land than that for lower quality land. This result is likely due to noisier land market signals for low-quality land, largely stemming from its greater variability in soil quality index and price paid per soil quality index point;

consequentially, cash rents and interest rates tend to play a larger role in determining experts' responses for lower quality land.

Our research has important implications for policymakers, agricultural professionals, and researchers of farmland markets in general, especially given the pervasive use of opinion surveys 
to gauge movements in land markets. Furthermore, expert opinion surveys like the Iowa survey analyzed in our paper are often the only source of land values at the county level. By providing empirical evidence that experts in land opinion surveys exhibit theoretically-consistent errorcorrection behavior with almost instantaneous adjustments to market signals, our analysis lends support to the accuracy of widely watched expert opinion surveys of land values conducted by land-grant universities and Federal Reserve banks. In addition, our findings on land quality shows that readers of these popular opinion surveys should pay more attention to results for high-quality land, and should exercise caution in interpreting and quoting results for low-quality land. Finally, our analysis focusing on experts' error-correction behavior suggests that devising estimation-correction techniques to adjust and improve the accuracy of land value surveys is a promising future research line, especially for low-quality land.

Of course, our research is not without limitations. First, because true farmland values are unobservable, we rely on noisy proxy variables to measure them, which may introduce errors in our estimation. To address this issue, our Bayesian approach employs an errors-in-variables approach, treating true values as unknown parameters with the proxy variables being their noisy signals or measurements. Second, although we use the median expert estimate as a proxy for the prevailing price in the base specification, experts do not directly observe the median response at the time of the survey. Instead, they could potentially see the publicly released land value estimates from the ISU survey, the RLI survey and, to a lesser extent, the FCS cropland prices. Various robustness checks show that our main conclusions of error-correction behavior with almost instantaneous adjustments remain robust to these alternative specifications and samples. Finally, the error-correction behavior uncovered in our research is arguably more transferrable for expert opinion surveys rather than producer or consumer surveys. 
Our analysis focuses on an expert opinion survey of farmland values. However, we conjecture that the error-correction behavior uncovered here is likely to characterize individual responses to a host of other opinion surveys. Our Bayesian approach may prove well suited for this task, as such surveys often involve non-stationary variables, unobservable "true" values, and/or short panels. This avenue of research is left for future studies to explore. Also worth pursuing in the future is the application of the present results to develop methods aimed at improving the accuracy of surveys in the presence of less-than-perfect error-correction behavior. 


\section{References}

Arellano, M., and S. Bond. (1991). "Some Tests of Specification for Panel Data: Monte Carlo Evidence and an Application to Employment Equations.” Review of Economic Studies 58:277-297.

Banerjee, A.V. (1992). “A Simple Model of Herd Behavior.” The Quarterly Journal of Economics 107:797-817.

Bigelow, D.P., J. Ifft, and T. Kuethe. 2020. "Following the Market? Hedonic Analysis Using Sales Prices versus Self-Reported Values.” Land Economics, forthcoming.

Borchers, A., J. Ifft, and T. Kuethe. (2014). "Linking the Price of Agricultural Land to Use Values and Amenities.” American Journal of Agricultural Economics 96:1307-1320.

De Boef, S., and L. Keele. (2008). “Taking Time Seriously.” American Journal of Political Science 52:184-200.

Dhaene, G., and K. Jochmans. (2016). "Likelihood Inference in an Autoregression with Fixed Effects.” Econometric Theory 32:1178-1215.

Fuller, W.A. (1987). Measurement Error Models. New York: John Wiley \& Sons.

Gelman, A., G. Goodrich, J. Gabry, and A. Vehtari. (2019). “R-squared for Bayesian Regression Models." The American Statistician 73(3):307-309.

Gelman, A., and D.B. Rubin. (1992). "Inference from Iterative Simulation Using Multiple Sequences." Statistical Science 7(4):457-72.

Hansen, K. (2018). “The Realtor Land Institute(RLI) Iowa Chapter Land Trends \& Value Survey." Ames, Iowa.

Harris, R.D.F., and E. Tzavalis. (1999). "Inference for Unit Roots in Dynamic Panels where the Time Dimension is Fixed." Journal of Econometrics 91:201-226. 
Kuethe, T., and J. Ifft. (2013). "The Information Content of Farmland Value Surveys." Agricultural Finance Review 73(1): 45-57.

Lancaster, T. (2002). “Orthogonal Parameters and Panel Data.” Review of Economic Studies 69:647-666.

Lence, S.H. (2001). "Farmland Prices in the Presence of Transaction Costs: A Cautionary Note." American Journal of Agricultural Economics 83:985-992.

—. (2014). "Farmland Prices: Is This Time Different?" Applied Economic Perspectives and Policy 36:577-603.

Nickell, S. (1981). "Biases in Dynamic Models with Fixed Effects." Econometrica 49(6):14171426.

Oppedahl, D. (2018). "Federal Reserve Bank of Chicago Land Values and Credit Conditions Survey." Federal Reserve Bank of Chicago.

Pickup, M., P. Gustafson, D. Cubranic and G. Evans. (2017). “OrthoPanels: An R Package for Estimating a Dynamic Panel Model with Fixed Effects Using the Orthogonal Reparameterization Approach.” The R Journal 9(1):60-76.

Plastina, A. (2018). “Cash Rental Rates for Iowa 2018 Survey.” Ag Decision Maker.

Sherrick, B., and P.J. Barry. (2003). "Farmland Markets: Historical Perspectives and Contemporary Issues.” In C. Moss and A. Schmitz, eds. Government Policy and Farmland Markets: The Maintenance of Farmer Wealth. Ames IA: Iowa State University Press, pp. 27-49.

Stan Development Team. (2019). "Stan Reference Manual. Version 2.19.0." Available at https://mc-stan.org/users/documentation/. 
Stinn, M., and M.E. Duffy. (2012). "What is the Precision of Land Survey Values?” Choices Magazine 27:1-4.

U.S. Department of Agriculture (USDA). (2019). "Farm Sector Balance Sheet 2019F.” In U.S. Farm Sector Financial Indicators, 2012-19F. USDA Economic Research Service.

Westerlund, J. (2007). “Testing for Error Correction in Panel Data.” Oxford Bulletin of Economics and Statistics 69:709-748.

Zhang, W. (2016). "How Tight is the Farmland Supply in Iowa?" Ag Decision Maker. October 2016.

Zhang, W., and Z. Beek. (2016). "Trends and Determinants of U.S. Farmland Values since 1910: Evidence from the Iowa Land Value Survey.” Farm Policy Journal 13:47-55.

Zhang, W., and M. Duffy. (2017). "Land Quality Perceptions in Expert Opinion Surveys: Evidence from Iowa.” Journal of the ASFMRA 2017:128-151.

Zhang, W., A. Plastina, and W. Sawadgo. (2018). "Iowa Farmland Ownership and Tenure Survey 1982-2017: A Thirty-five Year Perspective.” Iowa State University Extension and Outreach and Center for Agricultural and Rural Development, CARD Working Paper \#18-WP 580, available online at https://store.extension.iastate.edu/product/6492. 


\section{Figures}

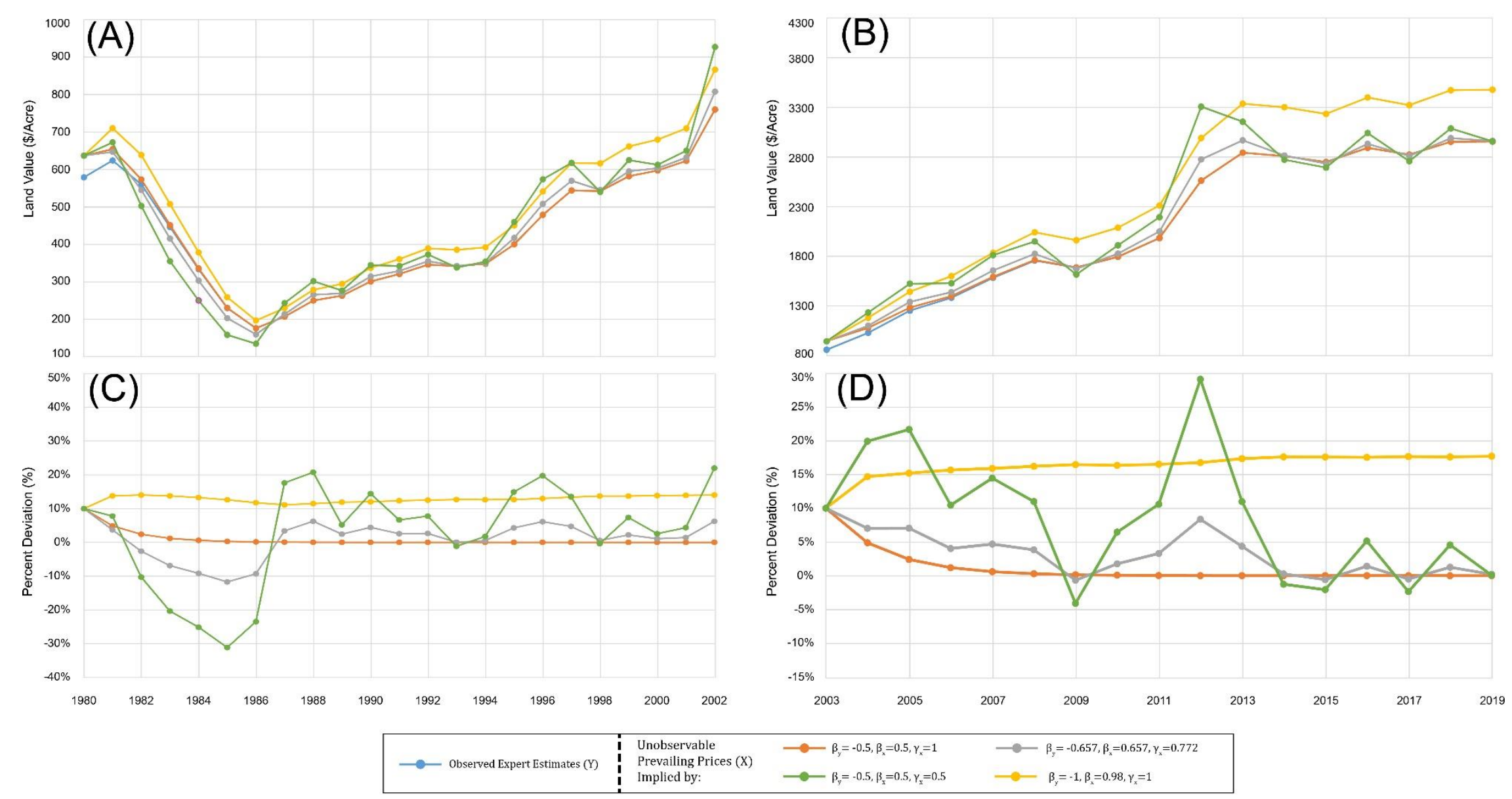

Figure 1. Average Expert Estimates to the ISU Survey and Unobservable Prevailing Prices implied by Different Parameters for Low

Quality Land in South Central Iowa, 1980 to 2019. 
Please select the ONE category that best describes your primary profession:

\begin{tabular}{|c|c|c|c|c|c|c|c|c|}
\hline & & $\begin{array}{l}\text { Professional } \\
\text { Farm }\end{array}$ & & & & Non-FSA & & \\
\hline Appraiser & $\begin{array}{c}\text { Ag Lender } \\
\mathrm{O}\end{array}$ & $\begin{array}{c}\text { Manager } \\
\mathrm{O}\end{array}$ & $\begin{array}{c}\text { Farmer } \\
0\end{array}$ & $\begin{array}{c}\text { Extension } \\
0\end{array}$ & $\begin{array}{c}\text { FSA } \\
0\end{array}$ & $\begin{array}{c}\text { Government } \\
\mathrm{O}\end{array}$ & $\begin{array}{c}\text { Sales } \\
0\end{array}$ & $\begin{array}{c}\text { Other } \\
\text { O }\end{array}$ \\
\hline
\end{tabular}

\section{Farmland values in your primary county as of November 1, 2015}

1. Land values for average-size farms in $<<$ field/county $>>$ County are:

\begin{tabular}{|c|c|c|c|c|}
\hline & $\begin{array}{c}\text { Your reported } \\
\text { values last year } \\
\text { (\$/acre) }\end{array}$ & $\begin{array}{l}\text { Your present } \\
\text { estimates } \\
\text { (\$/acre) }\end{array}$ & $\begin{array}{l}\text { Your estimated } \\
\text { average CSR1 }\end{array}$ & $\begin{array}{l}\text { Your estimated } \\
\text { average CSR2 }\end{array}$ \\
\hline High quality land & $<<$ Value_High $>>$ & & & \\
\hline $\begin{array}{l}\text { Medium quality } \\
\text { land }\end{array}$ & $\ll$ Value_Medium $>>$ & & & \\
\hline Low quality land & $\ll$ Value_Low $>$ & & & \\
\hline
\end{tabular}

Figure 2. Example Land Value Question from 2015 ISU Land Value Survey 
Table 1. Summary Statistics of Main Variables used to Perform Regressions

\begin{tabular}{|c|c|c|c|c|c|}
\hline Variable Description & Average & Std. Dev. & Min & Max & \# Obs \\
\hline High-quality land value estimate (\$/acre) & 7232 & 3335 & 756 & 20000 & 2880 \\
\hline Medium-quality land value estimate (\$/acre) & 5582 & 2633 & 610 & 16000 & 2880 \\
\hline Low-quality land value estimate (\$/acre) & 3880 & 1969 & 280 & 12000 & 2880 \\
\hline $\begin{array}{l}\text { CRD median of experts' value estimates for high- } \\
\text { quality land (\$/acre) }\end{array}$ & 7033 & 2854 & 2350 & 13200 & 2880 \\
\hline $\begin{array}{l}\text { CRD median of experts' value estimates for } \\
\text { medium-quality land (\$/acre) }\end{array}$ & 5286 & 2183 & 1500 & 10000 & 2880 \\
\hline $\begin{array}{l}\text { CRD median of experts' value estimates for low- } \\
\text { quality land (\$/acre) }\end{array}$ & 3584 & 1446 & 1100 & 7000 & 2880 \\
\hline $\begin{array}{l}\text { County median of experts' value estimates for high- } \\
\text { quality land (\$/acre) }\end{array}$ & 7055 & 3061 & 1825 & 19750 & 2861 \\
\hline $\begin{array}{l}\text { County median of experts'value estimates for } \\
\text { medium-quality land (\$/acre) }\end{array}$ & 5330 & 2354 & 1150 & 14750 & 2860 \\
\hline $\begin{array}{l}\text { County median of experts' value estimates for low- } \\
\text { quality land (\$/acre) }\end{array}$ & 3681 & 1697 & 800 & 10750 & 2859 \\
\hline $\begin{array}{l}\text { Number of years that respondents provided land } \\
\text { value estimates }\end{array}$ & 10 & 1 & 7 & 11 & 2880 \\
\hline Average cash rent at the CRD level (\$/acre) & 203 & 53 & 110 & 306 & 2880 \\
\hline Average cash rent at the county level (\$/acre) & 204 & 55 & 93 & 363 & 2880 \\
\hline $\begin{array}{l}\text { Chicago Fed January farmland loan interest rate }(\% \\
\text { per year) }\end{array}$ & 5.86 & 1.06 & 4.61 & 7.74 & 2880 \\
\hline $\begin{array}{l}\text { ISU published farmland values for high-quality } \\
\text { land at the CRD level as of Nov } 1^{\text {st }} \text { (\$/acre) }\end{array}$ & 7148 & 2830 & 2659 & 12890 & 2880 \\
\hline $\begin{array}{l}\text { ISU published farmland values for medium-quality } \\
\text { land at the CRD level as of Nov } 1^{\text {st }} \text { (\$/acre) }\end{array}$ & 5525 & 2209 & 1725 & 11011 & 2880 \\
\hline $\begin{array}{l}\text { ISU published farmland values for low-quality land } \\
\text { at the CRD level as of Nov } 1^{\text {st }} \text { (\$/acre) }\end{array}$ & 3824 & 1466 & 1252 & 7162 & 2880 \\
\hline $\begin{array}{l}\text { RLI published cropland values for high-quality land } \\
\text { at the CRD level as of September } 1^{\text {st }} \text { (\$/acre) }\end{array}$ & 7174 & 2922 & 2971 & 13337 & 2880 \\
\hline $\begin{array}{l}\text { RLI published cropland values for medium-quality } \\
\text { land at the CRD level as of September } 1^{\text {st }} \text { (\$/acre) }\end{array}$ & 5614 & 2147 & 2351 & 10303 & 2880 \\
\hline $\begin{array}{l}\text { ISU published cropland values for low-quality land } \\
\text { at the CRD level as of September } 1^{\text {st }} \text { (\$/acre) }\end{array}$ & 4016 & 1341 & 1919 & 6957 & 2880 \\
\hline $\begin{array}{l}\text { Mean sales prices for } 85 \% \text { tillable cropland at the } \\
\text { county level from Farm Credit Services of America } \\
\text { (\$/acre) }\end{array}$ & 7183 & 2495 & 1927 & 14982 & 2102 \\
\hline
\end{tabular}


Table 2. Posterior Distribution Estimates of Error-Correction Model with No Fixed Effects, Assuming that the Median of Experts' Opinions is a Proxy for the Unobservable True Land Value

\begin{tabular}{|c|c|c|c|c|c|c|}
\hline & \multicolumn{3}{|c|}{ True Value Proxy: Experts' County Median } & \multicolumn{3}{|c|}{ True Value Proxy: Experts' CRD Median } \\
\hline & High-Quality & Medium-Quality & Low-Quality & High-Quality & Medium-Quality & Low-Quality \\
\hline \multirow[t]{2}{*}{$y_{t-1}$} & $-.190 * * *(.010)$ & $-.189 * * *(.011)$ & $-.200 * * *(.012)$ & $-.190 * * *(.011)$ & $-.188 * * *(.011)$ & $-.199 * * *(.012)$ \\
\hline & {$[-.211,-.169]$} & {$[-.210,-.167]$} & {$[-.224,-.175]$} & {$[-.211,-.169]$} & {$[-.210,-.166]$} & {$[-.224,-.175]$} \\
\hline \multirow[t]{2}{*}{$x_{t-1}$} & $.231 * * *(.011)$ & $.217 * * *(.012)$ & $.200 * * *(.013)$ & $.231 * * *(.011)$ & $.216^{* * *}(.012)$ & $.200 * * *(.014)$ \\
\hline & {$[.211, .254]$} & {$[.194, .240]$} & {$[.174, .227]$} & {$[.210, .253]$} & {$[.195, .240]$} & {$[.173, .228]$} \\
\hline \multirow[t]{2}{*}{$\Delta x_{t}$} & $-.131 * * *(.029)$ & $-.159 * * *(.031)$ & $-.202 * * *(.032)$ & $-.132 * * *(.029)$ & $-.160 * * *(.031)$ & $-.203 * * *(.033)$ \\
\hline & {$[-.190,-.075]$} & {$[-.221,-.099]$} & {$[-.268,-.140]$} & {$[-.189,-.078]$} & {$[-.225,-.103]$} & {$[-.266,-.140]$} \\
\hline \multirow[t]{2}{*}{$\Delta y_{t-1}$} & $-.313 * * *(.021)$ & $-.326 * * *(.021)$ & $-.279 * * *(.021)$ & $-.312 * * *(.021)$ & $-.327 * * *(.021)$ & $-.280 * * *(.021)$ \\
\hline & {$[-.353,-.272]$} & {$[-.368,-.286]$} & {$[-.320,-.238]$} & {$[-.354,-.271]$} & {$[-.368,-.286]$} & {$[-.321,-.240]$} \\
\hline \multirow[t]{2}{*}{$\Delta x_{t-1}$} & $-.182 * * *(.031)$ & $-.259 * * *(.033)$ & $-.288 * * *(.035)$ & $-.182 * * *(.030)$ & $-.260 * * *(.033)$ & $-.288 * * *(.034)$ \\
\hline & {$[-.244,-.122]$} & {$[-.324,-.195]$} & {$[-.357,-.220]$} & {$[-.242,-.125]$} & {$[-.325,-.198]$} & {$[-.356,-.222]$} \\
\hline \multirow[t]{2}{*}{ Intercept } & $-.194(.147)$ & $-.067(.148)$ & $.158(.157)$ & $-.186(.152)$ & $-.062(.148)$ & $.157(.161)$ \\
\hline & {$[-.493, .088]$} & {$[-.359, .220]$} & {$[-.156, .467]$} & {$[-.483, .098]$} & {$[-.368, .222]$} & {$[-.162, .473]$} \\
\hline Long-Run & $1.223 * * *(.100)$ & $1.156 * * *(.101)$ & $1.006 * * *(.099)$ & $1.218 * * *(.103)$ & $1.153 * * *(.101)$ & $1.007 * * *(.102)$ \\
\hline Elasticity & {$[1.046,1.441]$} & {$[.974,1.370]$} & {$[.827,1.213]$} & {$[1.038,1.431]$} & {$[.973,1.372]$} & {$[.824,1.220]$} \\
\hline Std. Deviation & $.191 * * *(.003)$ & $.211 * * *(.003)$ & $.252 * * *(.004)$ & $.191 * * *(.003)$ & $.211 * * *(.003)$ & $.252 * * *(.004)$ \\
\hline of Residuals & {$[.185, .197]$} & {$[.205, .218]$} & {$[.244, .260]$} & {$[.185, .197]$} & {$[.205, .218]$} & {$[.244, .260]$} \\
\hline \multirow[t]{2}{*}{ Bayesian $\mathbf{R}^{2}$} & $.803(.006)$ & $.781(.007)$ & $.720(.008)$ & $.803(.006)$ & $.780(.007)$ & $.721(.009)$ \\
\hline & {$\left[\begin{array}{ll}.791 & .814\end{array}\right]$} & {$[.767, .794]$} & {$[.703, .737]$} & {$[.790, .814]$} & {$[.767, .794]$} & {$[.702, .737]$} \\
\hline Observations & 1,990 & 1,990 & 1,990 & 1,990 & 1,990 & 1,990 \\
\hline
\end{tabular}

$* * *(* *, *)$ Different from zero at the $1 \%(5 \%, 10 \%)$ level of significance, based on the respective $99 \%(95 \%, 90 \%)$ Credible Interval. Note: The table reports posterior means, posterior standard deviations (within parentheses), and $95 \%$ credible intervals [within brackets]. 
Table 3. Posterior Distribution Estimates of Error-Correction Model with Individual Expert Fixed Effects, Assuming that the Median of Experts' Opinions is a Proxy for the Unobservable True Land Value

\begin{tabular}{|c|c|c|c|c|c|c|}
\hline & \multicolumn{3}{|c|}{ County Level } & \multicolumn{3}{|c|}{ CRD Level } \\
\hline & High-Quality & Medium-Quality & Low-Quality & High-Quality & Medium-Quality & Low-Quality \\
\hline \multirow[t]{2}{*}{$y_{t-1}$} & $-.883 * * *(.038)$ & $-.829 * * *(.037)$ & $-.657 * * *(.035)$ & $-.882 * * *(.039)$ & $-.830 * * * *(.037)$ & $-.658 * * *(.036)$ \\
\hline & {$[-.956,-.808]$} & {$[-.901,-.756]$} & {$[-.728,-.590]$} & {$[-.959,-.805]$} & {$[-.901,-.759]$} & {$[-.726,-.588]$} \\
\hline \multirow[t]{2}{*}{$x_{t-1}$} & $.901 * * *(.059)$ & $.857 * * *(.058)$ & $.657 * * *(.053)$ & $.898 * * *(.060)$ & $.857 * * *(.058)$ & $.657 * * *(.052)$ \\
\hline & {$[.792,1.018]$} & {$[.755, .978]$} & {$[.557, .767]$} & {$[.794,1.027]$} & {$[.753, .979]$} & {$[.562, .766]$} \\
\hline \multirow[t]{2}{*}{$\Delta x_{t}$} & $.868 * * *(.047)$ & $.835 * * *(.047)$ & $.772 * * *(.046)$ & $.867 * * *(.048)$ & $.835^{* * *}(.047)$ & $.772 * * *(.046)$ \\
\hline & {$[.791, .969]$} & {$[.756, .937]$} & {$[.693, .870]$} & {$[.790, .972]$} & {$[.756, .935]$} & {$[.693, .871]$} \\
\hline \multirow[t]{2}{*}{$\Delta y_{t-1}$} & $.029(.026)$ & $.002(.027)$ & $-.049 *(.025)$ & $.028(.026)$ & $.003(.026)$ & $-.049 *(.026)$ \\
\hline & {$[-.023, .081]$} & {$[-.051, .054]$} & {$[-.100, .001]$} & {$[-.023, .079]$} & {$[-.049, .054]$} & {$[-.098, .003]$} \\
\hline \multirow[t]{2}{*}{$\Delta x_{t-1}$} & $-.008(.035)$ & $-.016(.037)$ & $.021(.036)$ & $-.008(.035)$ & $-.017(.037)$ & $.021(.037)$ \\
\hline & {$[-.074, .063]$} & {$[-.087, .054]$} & {$[-.049, .091]$} & {$[-.078, .061]$} & {$[-.088, .055]$} & {$[-.051, .092]$} \\
\hline Intercept & $\begin{array}{l}310 \text { individual } \\
\text { expert FEs }\end{array}$ & $\begin{array}{l}310 \text { individual } \\
\text { expert FEs }\end{array}$ & $\begin{array}{l}310 \text { individual } \\
\text { expert FEs }\end{array}$ & $\begin{array}{l}310 \text { individual } \\
\text { expert FEs }\end{array}$ & $\begin{array}{l}310 \text { individual } \\
\text { expert FEs }\end{array}$ & $\begin{array}{c}310 \text { individual } \\
\text { expert FEs }\end{array}$ \\
\hline Long-Run & $1.020 * * *(.049)$ & $1.033 * * *(.051)$ & $.999 * * *(.056)$ & $1.019 * * *(.050)$ & $1.033 * * *(.051)$ & $.999 * * *(.056)$ \\
\hline Elasticity & {$[.944,1.127]$} & {$[.954,1.147]$} & {$[.903,1.116]$} & {$[.946,1.129]$} & {$[.953,1.146]$} & {$[.905,1.120]$} \\
\hline Std. Deviation & $.151 * * *(.003)$ & $.174 * * *(.003)$ & $.218 * * *(.004)$ & $.151 * * *(.003)$ & $.174 * * *(.003)$ & $.218 * * *(.004)$ \\
\hline of Residuals & {$[.146, .157]$} & {$[.168, .180]$} & {$[.211, .226]$} & {$[.146, .156]$} & {$[.168, .180]$} & {$[.211, .226]$} \\
\hline \multirow[t]{2}{*}{ Bayesian $\mathbf{R}^{2}$} & $.853(.005)$ & $0.828(.006)$ & $.758(.009)$ & $.853(.005)$ & $0.828(.006)$ & $.758(.009)$ \\
\hline & {$[.842, .863]$} & {$[.815, .840]$} & {$[.740, .776]$} & {$[.842, .863]$} & {$[.815, .840]$} & {$[.740, .776]$} \\
\hline Observations & 1,989 & 1,989 & 1,989 & 1,989 & 1,989 & 1,989 \\
\hline
\end{tabular}

*** (**,*) Different from zero at the $1 \%(5 \%, 10 \%)$ level of significance, based on the respective $99 \%(95 \%$, $90 \%)$ Credible Interval.

Note: The table reports posterior means, posterior standard deviations (within parentheses), and $95 \%$ credible intervals [within brackets]. 
Table 4. Robustness Checks for FE ECMs Incorporating Orthogonal Components of Interest Rates and Rental Rates

\begin{tabular}{|c|c|c|c|}
\hline & & Proxy: Experts' Cou & \\
\hline & High-Quality & Medium-Quality & Low-Quality \\
\hline$y_{t-1}$ & $-.885 * * *(.038)$ & $-.833 * * *(.037)$ & $-.661 * * *(.035)$ \\
\hline & {$[-.958,-.810]$} & {$[-.905,-.761]$} & {$[-.725,-.590]$} \\
\hline$x_{t-1}$ & $.902 * * *(.060)$ & $.863 * * *(.058)$ & $.661 * * *(.053)$ \\
\hline & {$[.794,1.033]$} & {$[.758, .986]$} & {$[.562, .771]$} \\
\hline$\Delta x_{t}$ & $.867 * * *(.047)$ & $.837 * * *(.048)$ & $.773 * * *(.045)$ \\
\hline & {$[.789, .972]$} & {$[.758, .941]$} & {$[.695, .870]$} \\
\hline$\Delta y_{t-1}$ & $.029(.026)$ & $.004(.026)$ & $-.048 *(.026)$ \\
\hline & {$[-.022, .080]$} & {$[-.047, .055]$} & {$[-.101, .004]$} \\
\hline$\Delta x_{t-1}$ & $-.009(.035)$ & $-.018(.036)$ & $.021(.037)$ \\
\hline & {$[-.076, .060]$} & {$[-.089, .053]$} & {$[-.053, .093]$} \\
\hline$\perp$ rent $_{t-1}$ & $.009(.213)$ & $-.211(.239)$ & $-.452(.287)$ \\
\hline & {$[-.404, .419]$} & {$[-.675, .258]$} & {$[-1.022, .108]$} \\
\hline$\perp_{\text {interest }_{t-1}}$ & $-.845^{* * *}(.324)$ & $-.999 * * *(.345)$ & $-1.485^{* * *}(.373)$ \\
\hline & {$[-1.480,-.206]$} & {$[-1.683,-.308]$} & {$[-2.206,-.754]$} \\
\hline$\Delta \sqcup$ rent $_{t}$ & $.311^{* *}(.140)$ & $.335^{* *}(.155)$ & $.404^{* *}(.180)$ \\
\hline & {$[.037, .584]$} & {$[.031, .636]$} & {$[.048, .766]$} \\
\hline$\Delta \perp$ interest $_{t}$ & $-.550 * * *(.212)$ & $-.543 * *(.231)$ & $-.394(.282)$ \\
\hline & {$[-.950,-.125]$} & {$[-.991,-.084]$} & {$[-.948, .151]$} \\
\hline$\Delta \perp \operatorname{rent}_{t-1}$ & $.301 * *(.126)$ & $-.369 * * *(.136)$ & $.432 * *(.165)$ \\
\hline & {$[.058, .553]$} & {$[.102, .637]$} & {$[.111, .761]$} \\
\hline$\Delta \perp$ interest $_{t-1}$ & $.425 * *(.205)$ & $.594 * * *(.221)$ & $.818 * * *(.262)$ \\
\hline & {$[.017, .819]$} & {$[.165,1.040]$} & {$[.306,1.347]$} \\
\hline Intercept & 310 ind. expert FEs & 310 ind. expert FEs & 310 ind. expert FEs \\
\hline Long-Run & $1.019 * * *(.050)$ & $1.036 * * *(.052)$ & $.999 * * *(.056)$ \\
\hline Elasticity & {$[.944,1.129]$} & {$[.954,1.150]$} & {$[.905,1.120]$} \\
\hline Std. Deviation & $.150 * * *(.003)$ & $.173 * * *(.003)$ & $.216^{* * *}(.004)$ \\
\hline of Residuals & {$[.145, .156]$} & {$[.167, .179]$} & {$[.208, .223]$} \\
\hline Bayesian $\mathbf{R}^{2}$ & $.855(.005)$ & $.830(.006)$ & $.764(.009)$ \\
\hline & {$[.844, .865]$} & {$[.818, .842]$} & {$[.745, .781]$} \\
\hline Observations & 1,989 & 1,989 & 1,989 \\
\hline
\end{tabular}

$* * *(* *, *)$ Different from zero at the $1 \%(5 \%, 10 \%)$ level of significance, based on the respective $99 \%(95 \%, 90 \%)$ Credible Interval.
Note: The table reports posterior means, posterior standard deviations (within parentheses), and $95 \%$ credible intervals [within brackets]. 
Table 5. Robustness Checks for FE ECMs Assuming Unobservable True Land Values are Proxied by Average Sales Prices for 85\% Tillable Cropland Collected by the Farm Credit Services of America

\begin{tabular}{|c|c|c|c|}
\hline & True Value Pro & y Average Sales Pri & llable Cropland \\
\hline & High-Quality & Medium-Quality & Low-Quality \\
\hline$y_{t-1}$ & $-.896 * * *(.050)$ & $-.885 * * *(.048)$ & $-.830 * * *(.046)$ \\
\hline & {$[-.993,-.796]$} & {$[-.977,-.793]$} & {$[-.922,-.737]$} \\
\hline$x_{t-1}$ & $.754 * * *(.075)$ & $.733 * * *(.072)$ & $.658 * * *(.070)$ \\
\hline & {$[.610, .905]$} & {$[.595, .886]$} & {$[.527, .802]$} \\
\hline$\Delta x_{t}$ & $.599 * * *(.052)$ & $.543 * * *(.057)$ & $.454 * * *(.063)$ \\
\hline & {$[.501, .704]$} & {$[.437, .659]$} & {$[.334, .581]$} \\
\hline$\Delta y_{t-1}$ & $.081 * *(.033)$ & $.039(.031)$ & $.039(.031)$ \\
\hline & {$[.017, .146]$} & {$[-.021, .101]$} & {$[-.022, .099]$} \\
\hline$\Delta x_{t-1}$ & $.174 * * *(.049)$ & $.163 * * *(.049)$ & $.180 * * *(.054)$ \\
\hline & {$[.078, .270]$} & {$[.072, .259]$} & {$[.077, .286]$} \\
\hline Intercept & $\begin{array}{l}286 \text { individual } \\
\text { expert FEs }\end{array}$ & $\begin{array}{c}286 \text { individual } \\
\text { expert FEs }\end{array}$ & $\begin{array}{c}286 \text { individual } \\
\text { expert FEs }\end{array}$ \\
\hline Long-Run & $.841 * * *(.054)$ & $.827 * * *(.057)$ & $.793 * * *(.063)$ \\
\hline Elasticity & {$[.741, .956]$} & {$[.724, .946]$} & {$[.676, .922]$} \\
\hline Std. Deviation & $.180 * * *(.004)$ & $.196 * * *(.005)$ & $.236 * * *(.006)$ \\
\hline of Residuals & {$[.172, .188]$} & {$[.187, .205]$} & {$[.225, .247]$} \\
\hline Bayesian $\mathbf{R}^{2}$ & $.703(.017)$ & $0.660(.018)$ & $.573(.022)$ \\
\hline & {$[.668, .736]$} & {$[.623, .695]$} & {$[.528, .615]$} \\
\hline Observations & 1,312 & 1,312 & 1,312 \\
\hline
\end{tabular}

$* * *(* * * *)$ Different from zero at the $1 \%(5 \%, 10 \%)$ level of significance, based on the respective $99 \%(95 \%, 90 \%)$ Credible Interval. Note: The table reports posterior means, posterior standard deviations (within parentheses), and 95\% credible intervals [within brackets]. 


\section{Endnotes}

${ }^{1}$ Recently, several start-up companies, such as Granular AcreValue and FarmlandFinder, have tried to build showcase farmland sale prices. County assessors' offices increasingly utilize web interfaces such as Beacon and Vanguard, which allow current and future landowners and home buyers to search and view characteristics of residential houses and land parcels. However, this information is still very fragmented, limited to only the most recent sales, and lacks standardized formats.

${ }^{2}$ For simplicity, in the remainder of the study we will use the term "expert" to refer to a respondent to a farmland value survey.

${ }^{3}$ The technical details underlying Figure 1 will be described in the Model section.

${ }^{4}$ Nickell bias refers to the bias in the lagged dependent variable coefficient when dynamic fixed-effects panel models are estimated using a short time series and standard regression procedures.

${ }^{5}$ For a given set of variables $V$, variable $w$ can be decomposed as the sum $w=b V+\perp w(V)$, where $b$ is a conformable vector of coefficients and $\perp w(V)$ is orthogonal to $V$. Thus, $\perp w(V)$ can be interpreted as the additional information contained in $w$, conditional on $V$. By construction, the ordinary least squares estimator $b=\left(V^{\mathrm{T}} V\right)^{-1} V^{\mathrm{T}} w$ yields the desired decomposition. We use this fact to obtain the $\perp w$ regressors in ECM (8) as the residuals of an ordinary least squares regression of the corresponding variable $w$ on the set of ECM (5) regressors.

${ }^{6}$ An alternative approach to fitting regression (8) would be to use as regressors the original variables $w$ instead of their orthogonal components $\perp w$. It is straightforward to demonstrate that the residuals of the resulting regression are identical to the ones in $\operatorname{ECM}(8)\left(u_{i, t}\right)$, so that, as a whole, the explanatory power of the respective set of regressors is identical under the two alternative formulations. However, the regression with the original variables $w$ exhibits clear multi-collinearity problems, due to the fact that land values, rental rates, and interest rates are very highly correlated, which makes it difficult to test the hypothesis under investigation. Thus, and especially given that the present objective is to test whether rental rates and interest rates provide information conditional on true land values, regression (8) is better suited for the task at hand.

${ }^{7}$ The coefficients based on the expert responses $(y)$ and the true land values $(x)$ should be the same in ECMs (5) and (8), because by construction the regressors are orthogonal to the $\perp w$ variables. In essence, the proposed regression (8) is equivalent to postulating that the residual in ECM (5) behaves according to

$$
\begin{aligned}
& e_{i, t}=\beta_{\text {rent }} \perp \text { rent }_{c(i), t-1}+\beta_{\text {interest }} \perp \text { interest }_{t-1}+\gamma_{\text {rent }} \perp \Delta \text { rent }_{c(i), t}+\gamma_{\text {interest }} \perp \Delta \text { interest }_{t} \\
& +\sum_{n_{\text {rent }}=1}^{N_{\text {rent }}} \delta_{\text {rent }, n_{\text {rent }}} \perp \Delta r e n t_{c(i), t-n_{\text {rent }}}+\sum_{n_{\text {interest }}=1}^{N_{\text {inters }}} \delta_{\text {interest }, n_{\text {intersst }}} \perp \Delta \text { interest } t_{t-n_{\text {imerest }}}+u_{i, t .}
\end{aligned}
$$

${ }^{8}$ Note that the survey asks experts what the land value is, as opposed to what it should be. To illustrate the importance of this distinction, consider the hypothetical example of ECM (5) satisfying the ideal conditions $\left\{\alpha_{i}, \beta_{y}, \beta_{x}, \gamma_{x}, \delta_{y}, \delta_{x}\right\}=\{0,-1,1,1,0,0\}$, and that conditions at period $t$ are such that $\left(y_{i, t-1}-\right.$ $\left.x_{c(i), t-1}\right)=\Delta x_{c(i), t}=0$. Hence, the $i$ th expert's expected response innovation should be $\Delta y_{i, t}=0$, because her time- $(t-1)$ estimate was in equilibrium with the true land value, and there is no innovation in the true value. However, if the coefficients for the $\perp w$ variables in regression (8) are not zero, then the expert's expected response innovation need not be zero. For example, if $\perp \Delta \operatorname{rent}_{c(i), t}>0, \gamma_{\text {rent }}>0$, and all other $\perp w$ coefficients in (8) are zero, the expert's expected response innovation will be positive $\left(\Delta y_{i, t}=\gamma_{\text {rent }}\right.$ $\Delta$ rent $\left._{c(i), t}>0\right)$ rather than zero. It may be argued that this expert response is warranted because in this scenario the capitalization model indicates that the land value could be expected to adjust upward in the future in response to the orthogonal increase in land rents. The fallacy in this argument is that experts are asked to report their estimates of the true land value $x_{t}$, not what the land value should be according to the capitalization model (i.e., rent $t_{t}-$ interest $_{t}$ ), or any other theoretical valuation model for that matter. Finding coefficients $\left\{\beta_{\text {rent }}, \beta_{\text {interest }}, \gamma_{\text {rent }}, \gamma_{\text {interest }}, \delta_{\text {rent }}, \delta_{\text {interest }}\right\}$ significantly different from zero in (8) 
suggests that experts rely on information about rents and interest rates to gauge the value of land when they answer the survey.

${ }^{9}$ Even though ECMs are typically used in conjunction with non-stationary variables to represent (and test) cointegrating relationships, ECMs can also be used to represent the dynamics of stationary variables (De Boef and Keele 2008). However, in the case of classical econometrics, appropriate inference would require determining whether farmland values are stationary. This assertion is true because if farmland values are non-stationary, which is most likely to be the case (see, e.g., Lence 2001; 2014), standard classical inference methods no longer apply to conduct hypothesis testing in regressions like ECM (5). Thus, under a classical approach, one must first test whether farmland values are stationary or not. This can be done by means of the test proposed by Harris and Tzavalis (1999), which is designed for testing the null hypothesis of non-stationarity using panel data characterized by short time series and large cross sections. If farmland values are found to be non-stationary, the significance of the lagged land value coefficient can be tested using a homogeneous panel ECM cointegration test. A popular test for this purpose is the one introduced by Westerlund (2007), whose null hypothesis is no cointegration (i.e., that the lagged expert estimate coefficient equals zero).

${ }^{10}$ This feature proves useful here, because the long-run elasticity $-\beta_{x} / \beta_{y}$ is a nonlinear function of $\beta_{y}$. In this instance, the Bayesian approach allows us to compute the elasticity posterior in a straightforward manner (i.e., it is not necessary to use approximations like the delta method).

${ }^{11}$ Pickup et al. (2017) discuss alternative methods to account for the Nickell bias, including the popular Generalized Method of Moments (GMM) approach advocated by Arellano and Bond (1991), and provide evidence that Lancaster's method outperforms GMM. Similarly, Dhaene and Jochmans (2016) find that their approach performs better than GMM for a set of simulated scenarios.

${ }^{12}$ Recall that $x_{c(i), t}=x_{c(j), t}=x_{c, t}$ if experts $i$ and $j$ report for the same county $c$.

${ }^{13}$ The Bayesian $\mathrm{R}^{2}$, defined as the ratio of the variance of predicted values to the sum of the predicted variance and the expected error variance, estimates the share of variance explained for new data (Gelman et al., 2019).

${ }^{14}$ For the sake of completeness, table B.1 in Appendix B reports the analog of table 3 but without Nickell bias correction (i.e., excluding the last term from likelihood (A3)). Not correcting for the Nickell bias would mislead one to overestimate the speed at which experts correct their prior errors by over $20 \%$, and into inferring significant short-term dynamics.

${ }^{15}$ The $95 \%$ CIs for coefficient $\gamma_{x}$ in table 5 are entirely to the left of the corresponding posterior means in table 4, the upper bounds of the 95\% CIs for the long-run elasticities are smaller than one, and the $95 \%$ CIs for the lagged difference coefficient do not include zero. 